Check for updates

Cite this: Mater. Adv., 2020 1,1726

Received 24th May 2020,

Accepted 14th July 2020

DOI: 10.1039/d0ma00343c

rsc.li/materials-advances

\section{Oxygen, nitrogen co-doped molybdenum disulphide nanoflowers for an excellent antifungal activity $\dagger$}

\author{
Parbati Basu, ${ }^{a}$ Khushi Mukherjee, ${ }^{\mathrm{b}}$ Sudipta Khamrui, ${ }^{\mathrm{c}}$ Subharaj Mukherjee, ${ }^{\mathrm{d}}$ \\ Maudud Ahmed, ${ }^{d}$ Krishnendu Acharya, ${ }^{\mathrm{b}}$ Debamalya Banerjee, (D) ${ }^{\mathrm{c}}$ \\ Padinharu M. G. Nambissan (D) and Kuntal Chatterjee (D) *a
}

\begin{abstract}
The engineering of pristine low dimensional materials towards society-needed functionalities is the driving force for cultivation the field of nano-bio research. Herein, the molybdenum disulphide structure has been ornamented with nitrogen as well as oxygen atoms by a facile chemical route. This $\mathrm{O}, \mathrm{N}$ co-doped $\mathrm{MoS}_{2}$ nanoflower sample along with only-N-doped $\mathrm{MOS}_{2}$ samples were investigated structurally and chemically to ascertain the successful attachment of the dopant atoms and its associated changes in the structure. While positron annihilation spectroscopy demonstrates detailed defect pictures of these two variants with not much of a difference, the electron paramagnetic resonance displays the adequacy of $\mathrm{Mo}^{5+}$ defect states in $\mathrm{O}, \mathrm{N}$ co-doped $\mathrm{MoS}_{2}$ compared to the only$\mathrm{N}$-doped $\mathrm{MoS}_{2}$ sample. Moreover, this $\mathrm{O}, \mathrm{N}$ co-doped $\mathrm{MoS}_{2}$ exhibits outstanding antifungal activities towards two harmful fungal pathogens, namely, Alternaria alternata and Fusarium oxysporum, in dark as well as in light. $\mathrm{MoS}_{2}$, which otherwise showed no antifungal growth inhibition whatsoever, can thus be promoted to get exalted antifungal performance by gradual incorporation of nitrogen and oxygen atoms within the lattice of $\mathrm{MoS}_{2}$. This study establishes a new pathway to design better candidates for antifungal activity with lesser chances of antimicrobial resistance through suitable elemental engineering and is a prime need for our society to cope with the growing unforeseen dangers.
\end{abstract}

\section{Introduction}

In the wake of the vast catastrophe foisted on by the potato late blight-led Irish famine that went on to change the course of European history, ${ }^{1}$ it became truly imperative to develop rigorous pre-emptive measures to combat such invasive fungal infections in times to come. However, over the past centuries, the numerals of fungi or fungus-like oomycetes causing virulent diseases have only proliferated to pose a grave threat to the ecosystem. If not checked in time, this would not only put the ecological homeostasis at stake but also potentially

\footnotetext{
${ }^{a}$ Department of Physics, Vidyasagar University, Midnapore-721102, India. E-mail: kuntal@mail.vidyasagar.ac.in

${ }^{b}$ Molecular and Applied Mycology and Plant Pathology Laboratory, Department of Botany, University of Calcutta, Kolkata-700019, India

${ }^{c}$ Department of Physics, IIT Kharagpur, Kharagpur-721302, India

${ }^{d}$ Applied Nuclear Physics Division, Saha Institute of Nuclear Physics, Bidhannagar, Kolkata-700064, India

$\dagger$ Electronic supplementary information (ESI) available: Details of positron annihilation spectroscopy experiments, coincidence Doppler broadening spectroscopy experiments, fungitoxicity assays including growth inhibition test, TTC assay, fluorescence microscopy studies, comparison chart with other antifungal materials, and other useful results. See DOI: 10.1039/d0ma00343c
}

disrupt the food security, consequentially leading to a debilitated agricultural output. Furthermore, the efficacy of the presently available antifungal remedies in humans, namely, polyenes, azoles, flucytosine, and echinocandins has plateaued out ${ }^{2}$ and the incidences of invasive fungal infections in immunocompromised human population are incessantly on the rise. ${ }^{3}$ Ironically, the presence of several limitations, such as off-target toxicity, adverse interactions with other drugs, and the emergence of antifungal resistant strains have rendered these existent antifungals delimited in combating refractory and invasive fungal infections. The use of chemical-based pesticides, on the other hand, in the management of pre- and post-harvest decay and diseases in crops has been a long-standing issue of concern in the light of environmental pollution and safety in consuming foods ensuing out of toxic residuals of these fungicides. ${ }^{4}$ Therefore, it is essential to develop new environment-friendly antifungal nanomaterials with lower levels of toxicity and less chances of microbial resistance. In this matter, nanomaterial-based antifungals have evolved to be an exceptional alternative. ${ }^{5}$ The repertoire of nanomaterials explored so far in combating microbial invasions comprises of plasmonic nanoparticles, ${ }^{6}$ metal oxides, ${ }^{7,8}$ graphene oxide, ${ }^{9}$ graphene-based derivatives, ${ }^{10,11}$ and various 
hetero-structured nanocomposites. ${ }^{12-15}$ Of late, inspired by the grand success of graphene and graphene-based nanomaterials in antimicrobial activity, layered 2D materials, particularly molybdenum disulphide $\left(\mathrm{MoS}_{2}\right)$, have started to garner lot of interest in this field. However, till now, reports on the antifungal activities of $\mathrm{MoS}_{2}$ are strikingly very few in number.

Owing to the innumerable novel and intriguing properties arising due to quantum confinement in the third dimension, $\mathrm{MoS}_{2}$, a layered transition metal dichalcogenide, has found extensive applications in transistors, ${ }^{16}$ optoelectronic devices, ${ }^{17}$ spintronics, ${ }^{18}$ sensors, ${ }^{19}$ catalysts, ${ }^{20}$ photodetectors, ${ }^{21}$ energy storage devices, ${ }^{22}$ biomedicine, ${ }^{23}$ etc. In $\mathrm{MoS}_{2}$, a layer of Mo atoms is sandwiched between two layers of $\mathrm{S}$ atoms held by strong covalent bonding. ${ }^{24}$ However, due to the weak interlayer van der Waals force, mono- or few-layered $\mathrm{MoS}_{2}$ nanosheets with large specific surface area can be easily extracted from the bulk analogues by mechanical or chemical exfoliation methods. ${ }^{24}$ In addition, fluorescence or quenching of the fluorescence properties, which are entirely dependent on the thickness of the layers and the crystal structure, ${ }^{25,26}$ strong near-infrared region absorbance, and high photothermal quenching efficiency, ${ }^{27}$ have enabled the use of $\mathrm{MoS}_{2}$ in various biomedical applications including bio-sensing, bio-imaging, cancer therapy, drug delivery, antibacterial wound healing, and tissue regeneration. ${ }^{28,29}$ In spite of all these bio-related applications, the antifungal activities of $2 \mathrm{D} \mathrm{MoS}_{2}$ have remained largely unexplored till now. Although pristine bulk $\mathrm{MoS}_{2}$ has been hardly reported to exhibit any microbial inhibition, by controlling the thickness of the layers, lateral size, and crystal structure, the desired antimicrobial properties may be enforced within $\mathrm{MoS}_{2}$. Liu et al. demonstrated the photocatalytic bacterial disinfection of water by few-layered vertically aligned $\mathrm{MoS}_{2}$ nanofilms. ${ }^{30}$ Recently, $\mathrm{Xu}$ et al. showed that nanoflowers of $\mathrm{MoS}_{2}$ exhibited better antibacterial capabilities than the $\mathrm{MoS}_{2}$ nanosheets. ${ }^{31}$ Surface functionalization with hydrophobic ligands, ${ }^{32}$ antimicrobial peptides, ${ }^{33}$ and loaded antibiotics ${ }^{34}$ have been found to induce antimicrobial properties within the $\mathrm{MoS}_{2}$ nanosheets as well. Another fruitful strategy to impose antimicrobial behavior within the $\mathrm{MoS}_{2}$ nanosheets is to combine them with other antimicrobial nanomaterials such as silver and graphene oxide. ${ }^{35}$ Zhang et al. showed that chitosan and silver nanoparticlemodified $\mathrm{MoS}_{2}$ exhibited effectively large antifungal inhibition of Saccharomyces uvarum and Aspergillus niger. ${ }^{36}$ However, in all these cases, the bactericidal activities were observed to be engendered by light-dependent stimulations, which would therefore wholly restrain the scope of application of these antimicrobials for disinfection in implantable medical devices. In our previous work, ${ }^{37}$ we have shown that the defect sites, which are relatively abundant in $\mathrm{MoS}_{2}$, can be advantageously manipulated through nitrogen doping to make them suitable for the generation of reactive oxygen species (ROS) in dark as well, thereby invoking antifungal properties within it. Therefore, it was found that the advantageous design of the material in an appropriate way holds the key to attain success in developing powerful antifungals from $\mathrm{MoS}_{2}$ nanostructures; this is a relatively new and interesting field of study that requires intensive research work.
Here, in this work, we designed $\mathrm{MoS}_{2}$ nanostructures through suitable elemental doping and demonstrate how doping helps in significantly inducing high antifungal abilities in the $\mathrm{MoS}_{2}$ nanostructures, which would otherwise have no inhibitory effect on the fungal growth of phytopathogens. Moreover, we shall explain how the efficacy of the induced antifungal activity evolves on being co-doped with elements of differing electronegativities. Doping has always been a useful strategy to tune and reform functionalities such as electronic, magnetic, and catalytic. ${ }^{38-40}$ Interestingly, oxygen incorporation has been previously reported to have enhanced the catalytic performances in electrocatalytic hydrogen generation, ${ }^{39,40}$ supercapacitor, ${ }^{41}$ etc., but there are no reports on the doping by oxygen to achieve antifungal performances in $\mathrm{MoS}_{2}$ so far. Herein, we successfully doped oxygen in $\mathrm{MoS}_{2}$ in addition to N-doping through the facile hydrothermal method and studied its antifungal activities on Alternaria alternata and Fusarium oxysporum as version representatives of harmful fungal pathogens. Both these species have devastating effects not just on agricultural plants, leading to huge losses during the pre and post-harvest phases but also immunity-compromised humans and animals as well. Hence, the need to develop effectively potent antifungals to combat these fungal pathogens has grown to be an absolute necessity. Further, to get a comprehensive insight into the origin of the antifungal activities in these doped samples, various characterisation techniques were adopted and a possible mechanism and pathway leading to the fungal deaths has also been proposed.

\section{Experimental section}

\subsection{Materials}

All the chemicals and reagents were of analytical grade and purchased from Sigma Aldrich. They were used without any further purification.

\subsection{Synthesis of nitrogen-doped $\mathrm{MoS}_{2}$ and oxygen-nitrogen co-doped $\mathrm{MoS}_{2}$}

Here, in this work, nitrogen-doped molybdenum disulphide and oxygen-nitrogen co-doped $\mathrm{MoS}_{2}$ nanostructures, henceforth referred to as NMS and ONMS, respectively, were successfully synthesized through an optimised one-step hydrothermal method. On the other hand, bulk $\mathrm{MoS}_{2}$, henceforth referred to as MS in the text, of analytical grade was procured commercially from Sigma Aldrich. In order to prepare nitrogen-doped $\mathrm{MoS}_{2}$ (NMS), $0.5038 \mathrm{~g}$ of molybdenum trioxide $\left(\mathrm{MoO}_{3}\right)$ dissolved in $15 \mathrm{~mL}$ of DI water was added drop-wise to $0.8 \mathrm{M}$ aqueous solution of thiourea $\left(\mathrm{CSN}_{2} \mathrm{H}_{4}\right)$ and stirred vigorously on a magnetic stirrer for an hour. The resultant bluish white solution was then transferred to an autoclave of capacity $75 \mathrm{~mL}$ and heated in an oven at $180{ }^{\circ} \mathrm{C}$ for 48 hours. The black product so obtained was washed several times with de-ionised water and eventually dried at $60{ }^{\circ} \mathrm{C}$ to obtain the nitrogen-doped $\mathrm{MoS}_{2}$ powder (NMS). Further, oxygen-nitrogen co-doped $\mathrm{MoS}_{2}$ (ONMS) was prepared via a similar hydrothermal procedure, wherein the same precursor 
ratio and reaction temperature were maintained but the time of hydrothermal reaction was reduced to 18 hours.

\subsection{Characterisation}

In order to obtain the X-ray diffraction patterns, the synthesized powder samples were scanned in the $2 \theta$ range of $3^{\circ}-80^{\circ}$ with a step size of $0.02^{\circ}$ in a Rigaku Miniflex X-ray diffractometer having $\mathrm{CuK} \alpha$ radiation source of wavelength $1.5418 \AA$ A. A scanning electron microscope from Zeiss was used to analyse the morphology of the as-synthesized samples. Further, the transmission electron microscopy analysis of the obtained samples was done on a FEI-made Technai G2 20 Twin high resolution transmission electron microscope. The surface area measurements (BET) and pore size analysis was performed on the AutosorbiQ station from Quantachrome instruments. The Renishaw inVia Raman microscope was utilised to acquire the Raman spectra with an excitation wavelength of $532 \mathrm{~nm}$ at the room temperature. The X-ray photoelectron spectroscopy studies were recorded using a ULVAC-PHI5000 Versa Probe II spectrometer equipped with an $\mathrm{Al} \mathrm{K} \alpha$ radiation source having $1486.6 \mathrm{eV}$ of photon energy. The X-band electron paramagnetic resonance signals were recorded on the Bruker-made ELEXSYS E580 pulsed electron paramagnetic resonance spectrometer at room temperature. The microwave power for the measurements were fixed at $15 \mathrm{~mW}$. The details of positron annihilation spectroscopy (PAS) and coincidence Doppler broadening spectroscopy (CDBS) experiments are provided in the $\mathrm{ESI} \dagger$ (Section S1.1).

\subsection{Antifungal studies}

2.4.1 Materials. Alternaria alternata (NFCCI-3151) and Fusariumoxysporum (NFCCI-2360), used as the model fungal pathogens, were purchased from Agharkar Research Institute, Pune, India.

2.4.2 Maintenance of the fungal culture. The fungal pathogens were maintained in Potato Dextrose Agar (PDA) slants and grown at $30{ }^{\circ} \mathrm{C}$ temperature for 7 days. The fungal cultures were preserved at $4{ }^{\circ} \mathrm{C}$ and grown freshly before every experiment.

2.4.3 Fungitoxicity assay. The details of the fungitoxicity assay include the growth inhibition test, Triphenyl Tetrazolium Chloride (TTC) assay, scanning electron microscopy analysis, and fluorescence microscopy studies to detect fungal cell death and ROS generation is given in the $\mathrm{ESI} \dagger$ (Sections S1.2.1-S1.2.4).

\section{Results and discussion}

Here, in this work, the incorporation of only nitrogen atoms in the case of NMS and both oxygen and nitrogen in the case of ONMS as dopants within the lattice was optimised and achieved through sustainable control on the time over which the reaction took place. $\mathrm{NH}_{3}$ evolution as a by-product in the thermal decomposition of thiourea accounts for the nitrogen atoms being doped within the $\mathrm{MoS}_{2}$ lattice, which according to our previous study, are most likely to occupy the S-sites in the Mo-rich limit. ${ }^{37}$ However, in order to ensure the incorporation of oxygen into the $\mathrm{MoS}_{2}$ lattice in addition to the nitrogen atoms, the reaction time had to be substantially brought down to 18 hours. The reduced reaction time effectively rendered the reaction process insufficient for the complete conversion of $\mathrm{MoO}_{3}$ to $\mathrm{MoS}_{2}$. Hence, traces of unsaturated Mo-O bonds, inherited from the $\mathrm{MoO}_{3}$ precursor, remained within the lattice of $\mathrm{MoS}_{2}$ to ensure the presence of oxygen atoms within the $\mathrm{MoS}_{2}$ lattice. Previously, in a similar kind of synthetic procedure, Xie et al. had incorporated oxygen in $\mathrm{MoS}_{2}$ nanosheets by controlling the crystallisation process through temperature variation in order to achieve enhanced electro-catalytic activity in the hydrogen evolution reaction. ${ }^{40}$ Besides, in earlier works, surface oxidation achieved through various other methods such as exposure to high energy RF oxygen plasma, ${ }^{42}$ remote plasma, ${ }^{43}$ $1.5-5 \mathrm{eV}$ atomic oxygen flux, ${ }^{44} \mathrm{UV}$-ozone exposure, ${ }^{45}$ and annealing in a high temperature oxygen environment, ${ }^{46}$ were used. These are rather harsh methods and resulted in non-uniform lateral oxidation and in-depth lattice etching. In contrast, the strategy adopted in this present work is a far more simplistic approach, resulting in a high percentage of oxygen doping.

In order to determine the morphology and microstructure of the as-grown doped $\mathrm{MoS}_{2}$ samples, scanning electron microscopy (SEM) and transmission electron microscopy (TEM) were conducted. The SEM images, as shown in Fig. 1(a) and (b), reveal almost similar kinds of flower-like morphology for both NMS and ONMS. However, in the case of ONMS, the $\mathrm{MoS}_{2}$ nanosheets, which assembled themselves into a flower-like motif, are apparently seen to be considerably thickened and also have surface roughness. Further, in ONMS, the edges of the nanosheets are obscure, suggesting insufficient crystallisation due to reduced reaction time. On the contrary, the NMS sheets have relatively sharper edges and reveal better crystallinity. The high magnification TEM images [Fig. 1(c) and (d)] further indicate the assemblage of nanosheets in a flower-like manner, as obtained from scanning electron microscopy. The high-resolution transmission electron microscopy (HRTEM) images reveal an enlarged $d$-spacing of $\sim 0.855 \mathrm{~nm}$ ONMS compared to that of $0.66 \mathrm{~nm}$ [Fig. 1(e) and (f)]. In congruence with the earlier studies, ${ }^{39-41}$ this enlargement in the interplanar $d$-spacing can be attributed to the incorporation of oxygen in the $\mathrm{MoS}_{2}$ lattice. However, the $d_{002}$ value calculated from the fringes of NMS agrees well with the reported values of $2 \mathrm{H}-\mathrm{MoS}_{2} \cdot{ }^{37}$ In addition to this, X-ray diffraction (XRD) studies were carried out on the synthesized samples in order to figure out the crystal structure. The XRD pattern for NMS, as shown in Fig. 2(a), matches well with the data of $2 \mathrm{H}-\mathrm{MoS}_{2}$ (JCPDS card no. 87-2416). The interplanar spacing $\left(d_{002}\right)$ calculated from the XRD peak value agrees well with those calculated from the HRTEM fringes. However, as evident from Fig. 2(a), two new peaks at $10.28^{\circ}$ and $16.87^{\circ}$ were seen to emerge for ONMS. The $d$-spacing calculated for these two peaks is $0.861 \mathrm{~nm}$ and $0.524 \mathrm{~nm}$, respectively. This diploidic relation corresponding to the interplanar spacing unambiguously suggests that a new lamellar structure with an expanded interplanar spacing has been formed. This possibly accounts for the thickening of the nanosheets in ONMS as observed from the SEM images. The simulated XRD pattern for oxygen incorporated $\mathrm{MoS}_{2}$ nanosheets 
a)

b)

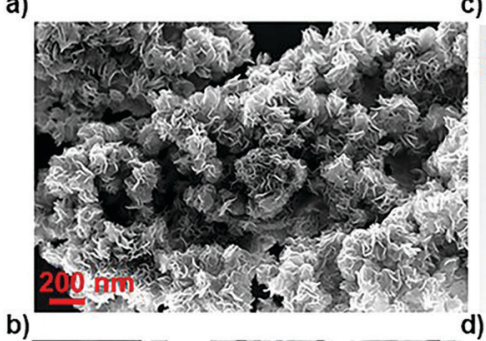

c)

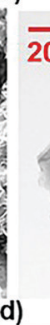

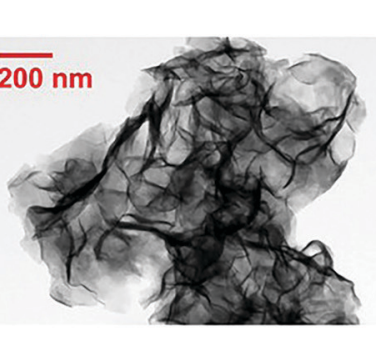

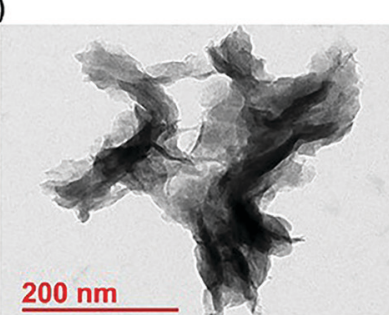

e)

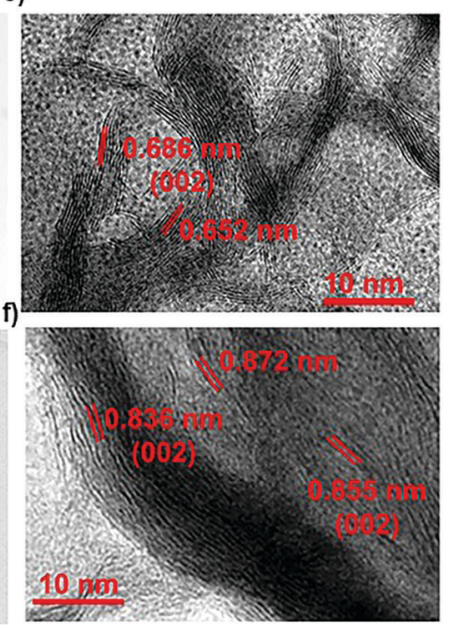

Fig. 1 (a and b) Scanning electron microscopy images of (a) NMS, and (b) ONMS. (c and d) Transmission electron microscopy images of (c) NMS and (d) ONMS. (e and f) HRTEM images of (e) NMS and (f) ONMS, showing enlarged interplanar spacing.

taken from Xie et al. is inserted in Fig. 2(a) for comparison and is seen to match with the XRD peaks of ONMS in the low angle region. ${ }^{40}$ Nonetheless, the XRD peaks in ONMS corresponding to the (102) and (110) planes match with those of $2 \mathrm{H}-\mathrm{MoS}_{2}{ }^{37}$ The surface area and porosity of the synthesized samples were ascertained by the adsorption-desorption studies of nitrogen. The isotherm plots for ONMS and NMS are shown in Fig. S1 (ESI $\dagger$ ) and suggest the presence of mesopores within the samples. Applying Brunauer-Emmett-Teller (BET) analysis, the surface areas for ONMS and NMS were calculated to be $6.507 \mathrm{~m}^{2} \mathrm{~g}^{-1}$ and $6.484 \mathrm{~m}^{2} \mathrm{~g}^{-1}$, respectively. Further, the average pore radius in ONMS was $38.468 \AA$ with a total pore volume of $1.252 \times 10^{-2} \mathrm{cc} \mathrm{g}^{-1}$ for the pores of radius less than $144.9 \mathrm{~nm}\left(P / P_{0}=0.993\right)$. In contrast, NMS showed a marginally increased average pore radius of $40.78 \AA$ with a total pore volume of $1.332 \times 10^{-2} \mathrm{cc} \mathrm{g}^{-1}$ for the pores of radius less than $210.4 \mathrm{~nm}\left(P / P_{0}=0.995\right)$.

Raman spectroscopy was further employed to look into the modes of vibration in the samples in greater details. In Fig. 2(b), the obtained Raman spectra for NMS and ONMS are shown. In the $2 \mathrm{H}$ representative of bulk $\mathrm{MoS}_{2}$, the two Raman active modes are the $\mathrm{E}_{2 \mathrm{~g}}^{1}$ mode resulting from the oppositely directed in-plane vibration of the Mo and $\mathrm{S}$ atoms and the $\mathrm{A}_{1 \mathrm{~g}}$ mode as a consequent of their out-of-plane opposite vibrations. ${ }^{47}$ The $\mathrm{E}_{2 \mathrm{~g}}^{1}$ and $\mathrm{A}_{1 \mathrm{~g}}$ modes occur nearly at $382 \mathrm{~cm}^{-1}$ and $407 \mathrm{~cm}^{-1}$ in the bulk form of $\mathrm{MoS}_{2} \cdot{ }^{47}$ However, in the case of both ONMS and NMS, the $\mathrm{E}_{2 \mathrm{~g}}^{1}$ mode is red-shifted by $\sim 4.5 \mathrm{~cm}^{-1}$ in comparison to the reported values of 6-layered $\mathrm{MoS}_{2}{ }^{47}$ This red shift possibly ensues from the lattice distortion invoked by oxygen and nitrogen doping in the samples. This is further reaffirmed by the disorder-prompted symmetry breaking of the $E_{2 g}^{1}$ mode, causing it to split into the ${ }_{2 g}^{1+}$ and $E_{2 g}^{1-}$ components, ${ }^{37,48}$ as shown in Fig. S2 (ESI $\dagger$ ). Meanwhile, the $A_{1 g}$ Raman mode is red-shifted in ONMS by almost $3 \mathrm{~cm}^{-1}$ relative to the 6-layered $\mathrm{MoS}_{2}$, which is more pronounced than the $\sim 1 \mathrm{~cm}^{-1}$ red-shift in the case of NMS. Moreover, the emergence of Raman peaks at $284.32 \mathrm{~cm}^{-1}, 331.18 \mathrm{~cm}^{-1}$, and $218.75 \mathrm{~cm}^{-1}$, assigned to the $\mathrm{B}_{2 \mathrm{~g}}, \mathrm{~B}_{1 \mathrm{~g}}$, and $\mathrm{A}_{\mathrm{g}}$ modes of vibration for the Mo-O bonds, reconfirms the successful doping of oxygen in ONMS. ${ }^{40,49}$

In order to get further insight about the chemical constitution of the as-synthesized samples, X-ray photoelectron spectroscopy (XPS) was performed on the doped $\mathrm{MoS}_{2}$ samples. The multi-component core level XPS spectra was deconvoluted with the help of mixed Gaussian-Lorentzian product function alongside a Shirley background correction using the CASAXPS software. In NMS, the deconvolution of the core Mo 3d region resulted in two doublets, one between $\sim 228.6 \mathrm{eV}$ and $231.74 \mathrm{eV}$, and the other between $\sim 228.9 \mathrm{eV}$ and $232.04 \mathrm{eV}$, as seen from Fig. 3(a). The lower binding energy doublet corresponds to the Mo $3 \mathrm{~d}_{5 / 2}$, and Mo $3 \mathrm{~d}_{3 / 2}$ core levels in $\mathrm{MoS}_{2}$, i.e., $\mathrm{Mo}^{4+}$ and $\mathrm{S}^{2-}$ charge states arising out of S-Mo charge transfer. ${ }^{48,50}$ The corresponding peaks in the core level $\mathrm{S} 2 \mathrm{p}$ spectra for the $\mathrm{S}^{2-}$ charge state are seen at $161.61 \mathrm{eV}$ and $162.74 \mathrm{eV}$. The other doublet in the Mo 3d region, at binding energies higher than the Mo-S peak by $\sim 0.3 \mathrm{eV}$, which may be attributed to the formation of $\mathrm{Mo}-\mathrm{N}$ bonds in agreement with the previous reports. ${ }^{48}$ This is further affirmed by the presence of the peak at $397.59 \mathrm{eV}$ in the $\mathrm{N} 1 \mathrm{~s}$ region [Fig. 3(c)], which essentially signifies Mo-N bond formation. ${ }^{37,48,51}$ Also, the $\mathrm{N}$ 1s region is partially overlapped by the core level Mo $3 \mathrm{p}_{3 / 2}$ region and the peak at $394.47 \mathrm{eV}$ originates from the Mo-S bonds in $\mathrm{MoS}_{2} \cdot{ }^{37,48}$ The remaining peak at $\sim 401 \mathrm{eV}$ arises because of the strongly chemisorbed ammonia. ${ }^{37,51}$ A small hump near $232.95 \mathrm{eV}$ in the Mo 3d region of NMS may be ascribed to superficial oxidation of the synthesized NMS sample instead of any Mo-O bond formation. A similar peak at $\sim 233.4 \mathrm{eV}$ is present in the Mo 3d region of ONMS as well. The absence of any Mo-O peaks in the O 1s region of NMS further substantiates our claim of surface oxidation. The peaks at $531.26 \mathrm{eV}, 532.3 \mathrm{eV}$, and $533.77 \mathrm{eV}$ in the $\mathrm{O}$ 1s region of NMS, as shown in Fig. S3(b) (ESI $\dagger$ ), are primarily due to oxygen in the $-\mathrm{OH}$ radical (possibly physisorbed $\mathrm{H}_{2} \mathrm{O}$ ), $\mathrm{O}_{2}$ adsorbed from the atmosphere, and physisorbed/chemisorbed $\mathrm{H}_{2} \mathrm{O}$ at the surface respectively. ${ }^{52-54}$ Further, the peaks at $226.17 \mathrm{eV}$ and $224.87 \mathrm{eV}$ in 

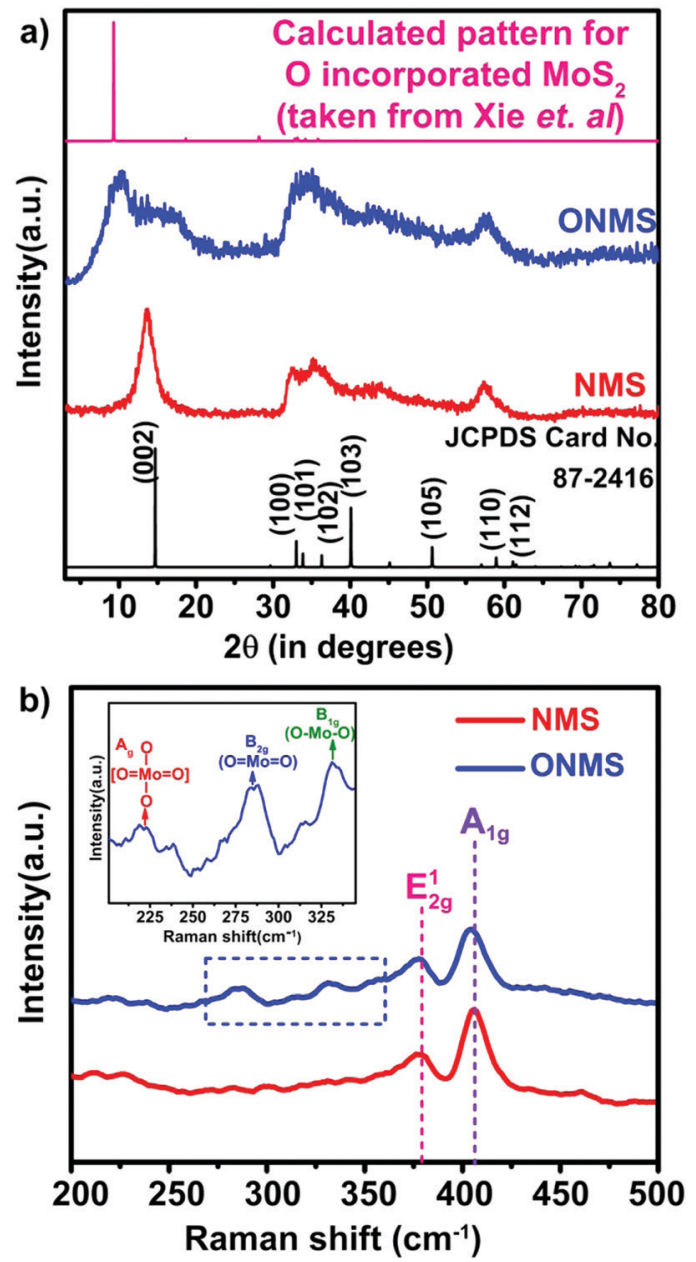

Fig. 2 (a) X-ray diffraction patterns NMS and ONMS. The standard pattern for commercially procured $2 \mathrm{H}-\mathrm{MoS}_{2}$ and the calculated pattern for oxygen-incorporated $\mathrm{MoS}_{2}$ lattice (taken from Xie et al.) ${ }^{40}$ is given as the reference. (b) Raman spectra for NMS and ONMS. The dotted blue rectangle emphasizes the development of the Mo-O bond in ONMS The inset shows the presence of three Raman peaks assigned to different Mo-O bond formations in ONMS under zoomed condition.

the overlapped S 2s region arise because of Mo-S bonding in $\mathrm{MoS}_{2}$ and the cluster complexes of Mo and S, respectively. ${ }^{55}$ On the other hand, in ONMS, the doublets in the Mo 3d region arising out of Mo-S and Mo-N bond formation are shifted down to lower binding energies, as is revealed from Fig. 3(a). The $S$ p peaks associated with the $\mathrm{S}^{2-}$ state are also downshifted to lower energy values, as shown in Fig. 3(b). The intensity of the doublet arising out of Mo-N bonding, however, decreased for ONMS, suggesting reduced N-doping compared to NMS. In addition to this, another doublet is seen to arise between $229.72 \mathrm{eV}$ and $232.73 \mathrm{eV}$ in Mo $3 \mathrm{~d}$ region for ONMS. According to earlier reports, ${ }^{56}$ this doublet emerges because of the incorporation of oxygen in $\mathrm{MoS}_{2}$ to form a $\mathrm{MoS}_{2-x} \mathrm{O}_{x}$-like phase with the $\mathrm{Mo}^{5+}$ charge state. Further, the peak at $399.162 \mathrm{eV}$ in the overlapped $\mathrm{N}$ 1s region of ONMS [Fig. 3(c)], in addition to the little Mo-N peak at $397.44 \mathrm{eV}$, emerges due to the introduction of Mo-O bonds. ${ }^{57}$ Also, the peak at $530.2 \mathrm{eV}$ in the core level of the $\mathrm{O} 1 \mathrm{~s}$ region for ONMS reaffirms the presence of Mo-O bonds ${ }^{41}$ in it, as evident from Fig. S3(a) (ESI $\dagger$ ). However, the other peaks at $531.10 \mathrm{eV}$ and $532.06 \mathrm{eV}$ in the $\mathrm{O} 1 \mathrm{~s}$ region are due to the $-\mathrm{OH}$ radical and adsorbed oxygen. Hence, it can be inferred that in addition to being nitrogen-doped, oxygen has also been successfully incorporated into the $\mathrm{MoS}_{2}$ lattice of ONMS through substitutional doping rather than surface oxidation. The downshift of the doublets in the core level Mo 3d region of ONMS, as mentioned before, may be explained to occur as a result of the Fermi level realignment between $\mathrm{MoS}_{2}$ and $\mathrm{MoO}_{x}$, giving rise to upward band bending. ${ }^{43,58}$ The peak at $235.29 \mathrm{eV}$ in the Mo $3 \mathrm{~d}$ region suggests the presence of residual unreacted $\mathrm{MoO}_{3}$ for ONMS. ${ }^{37}$ The $\mathrm{S} 2 \mathrm{p}$ region for ONMS [Fig. 3(b)] on being deconvoluted resulted primarily into two doublets, out of which, one with its peaks at $161.17 \mathrm{eV}$ and $162.41 \mathrm{eV}$ arises because of the $\mathrm{S}^{2-}$ state of $\mathrm{MoS}_{2}$ and the other between $162.86 \mathrm{eV}$ and $164 \mathrm{eV}$ results because of the $\mathrm{S}_{2}{ }^{2-}$ state, which is normally seen to accompany the $\mathrm{Mo}^{5+}$ charge state. ${ }^{50,59}$ The quantitative analysis of the survey spectra, as shown in Fig. S4 (ESI $\dagger$ ), reveals $15.97 \%$ of oxygen doping as well as $4.89 \%$ of $\mathrm{N}$-doping in ONMS whereas there is $6.38 \%$ nitrogen-doping in NMS.

The X-band electron paramagnetic resonance (EPR) study conducted on the doped $\mathrm{MoS}_{2}$ samples revealed the presence of a weak broad signal at $\sim g=2.00$ in both the ONMS and NMS samples, as shown in Fig. 4(a). This signal in the EPR spectrum,
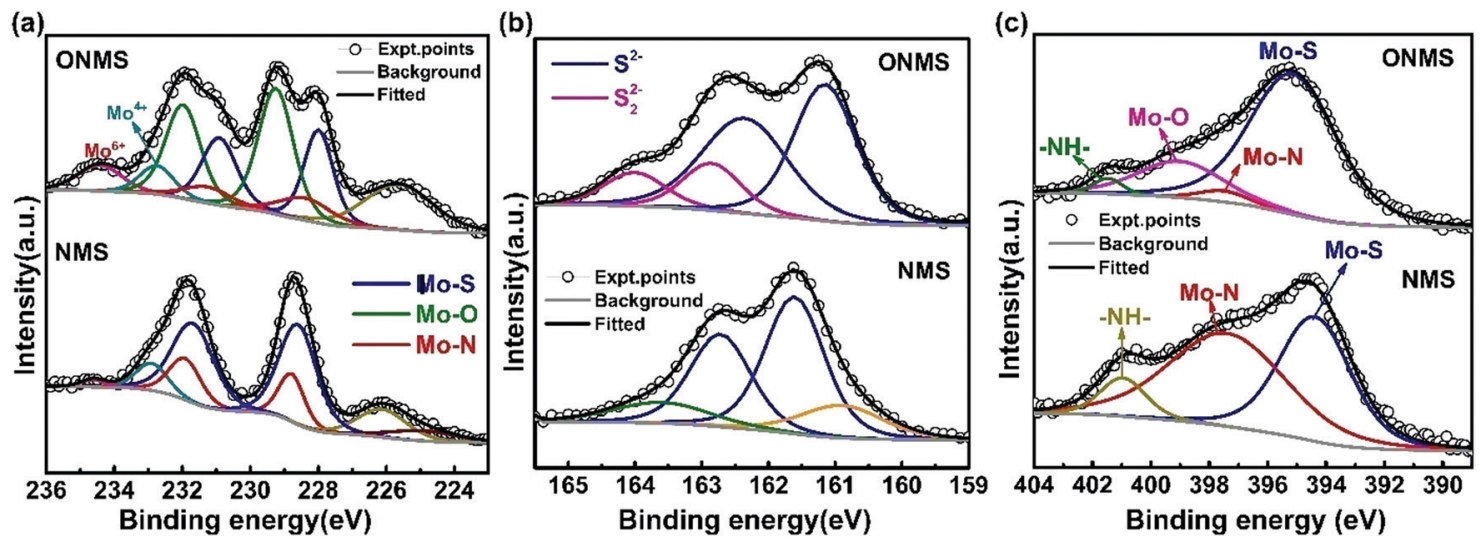

Fig. 3 High resolution $X$-ray photoelectron spectrum from the (a) Mo $3 d$, (b) $S 2 p$, and (c) N 1s regions of ONMS and NMS. 
according to previous studies, arises due to sulphur-coordinated (thio-Mo ${ }^{5+}$ ) defects in $\mathrm{MoS}_{2}{ }^{60}$ The intensities of the $\sim g=2.00$ signal in both ONMS and NMS appear to be almost equal at a glance, indicating that there is not much of a difference in the coordination environment of the sulphur-moderated defect sites or their relative abundance. However, the presence of a peak at $g=1.9364$ [Fig. 4(a)] for ONMS makes it different from NMS as far as the oxygen-coordinated (oxo- $\mathrm{Mo}^{5+}$ ) defect states are concerned. According to earlier reports, the $g=1.9364$ line arises because of the hyperfine interaction of the unpaired electrons in the $\mathrm{Mo}^{5+}$ state, which consists of a molybdenyl centre, i.e., a short $\mathrm{Mo}=\mathrm{O}$ bond surrounded by oxygen ligands in distorted octahedral or square-pyramidal symmetry. ${ }^{50,61}$ Therefore, this strongly evinces the successful doping of oxygen atoms throughout the sample in the case of ONMS.

In order to get a comprehensive insight into the nature of the defect states, as revealed by the EPR spectra, positron annihilation spectroscopy (PALS) was carried out on both ONMS and NMS. The peak-normalised positron lifetime spectra of ONMS and NMS are given in Fig. 4(b). Not much of a difference is observed between the two samples, although the resolved positron lifetimes and intensities (showed in Table 1) are more informative and sharper in detail as far as the sizes and concentrations of the defect structures present in the samples are concerned.

The observation of a third positron(ium) lifetime component $\tau_{3}$ is quite unnatural for materials with relatively higher electron densities like as $\mathrm{MoS}_{2}$ since it normally arises due to the formation of positronium (Ps), which is the metastable bound state of an electron and a positron. It can happen in nanocrystalline materials where large intercrystalline boundaries exist, which are characterised by the significantly reduced electron density compared to that of the bulk. However, in this case, the efforts to analyse the spectra with two lifetime components yielded poor reduced $\chi^{2}$ fit. The three-component analysis also gave scattered values of the third component of lifetime $\tau_{3}$ and relative intensity $I_{3}$. So, the analyses were done by fixing $\tau_{3}$ to $0.55 \mathrm{~ns}$, which incidentally is the highest possible positron lifetime when it is annihilated from the nanocrystalline surfaces. From the results shown in Table 1, the first conclusion that can be drawn is the existence of two types of structural defects, one arising out of the atomistic defects or crystalline vacancies and the other as porous type defects in the free spaces within the molecular assemblage, as revealed by the BET surface analysis. The positron lifetimes $\tau_{2}$ and $\tau_{3}$ represent these defects and the magnitudes of these lifetimes also indicate the nature and sizes of the defects. For example, the lifetime of the positrons annihilating from the free state (i.e., not trapped in any defect and diffusing freely through the inter-atomic region) is calculated for both the cases from the relation

$$
\frac{1}{\tau_{1}}=\frac{1}{\tau_{\mathrm{b}}}+\kappa
$$

where the positron trapping rate

$$
\kappa=\frac{I_{2}}{I_{1}}\left(\frac{1}{\tau_{\mathrm{b}}}-\frac{1}{\tau_{2}}\right)
$$

and the value of $\tau_{\mathrm{b}}$ so obtained helps to identify the atomistic defects present in the samples. The difference $\tau_{2}-\tau_{\mathrm{b}}$, which is $0.1112 \mathrm{~ns}$ for the ONMS sample and $0.1147 \mathrm{~ns}$ for the NMS sample, corresponds to the defects of divacancy type $\left(\tau_{2}-\tau_{\mathrm{b}}=0.103 \mathrm{~ns}\right)$ and trivacancy type $\left(\tau_{2}-\tau_{\mathrm{b}}=0.1190 \mathrm{~ns}\right)$ clusters, more or less in equal proportions in ONMS and tending more towards the latter in NMS (Table 1). The intensity $I_{2}$ differs by about $4 \%$ in the NMS sample, indirectly hinting at a slightly larger defect concentration in it. The defect concentration is calculated from the relation $\kappa=\mu C$ where the specific positron trapping rate $\mu$ is $0.590 \mathrm{~ns}^{-1}$ for divacancies and $0.770 \mathrm{~ns}^{-1}$ for trivacancies. For tetravacancy clusters, $\mu$ is $0.970 \mathrm{~ns}^{-1}$ and so on. ${ }^{62}$ The estimated defect concentrations are also given in Table 1 . The slightly lower value in the ONMS sample is perhaps an indication of the occupancy of some of these defects by oxygen atoms, although the process was not successful enough to reduce their number significantly.

The coincidence Doppler broadening spectra (CDBS), as shown in Fig. 4(c), also give results that are consistent with those obtained from the positron annihilation lifetime measurements. The peak at $P_{\mathrm{L}}=9.1 \times 10^{-3} m_{0} c$ that arises from positron annihilation with the 4 p electrons of Mo is stronger in the case of NMS when compared to that of ONMS. This again proves that the defect concentration in NMS is slightly on the higher side. The calculated defect concentration, as obtained (a)

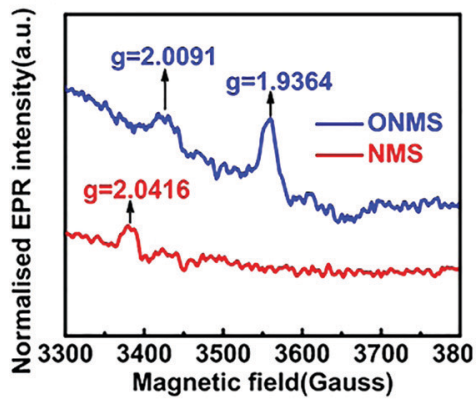

(b)

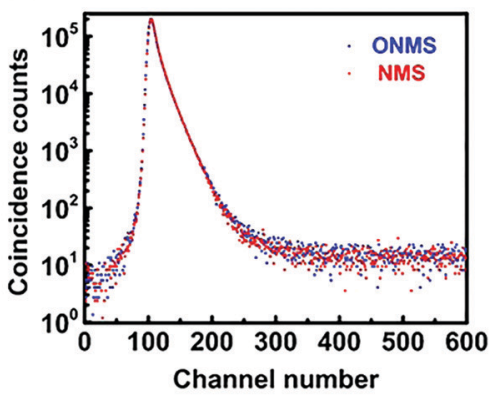

(c)

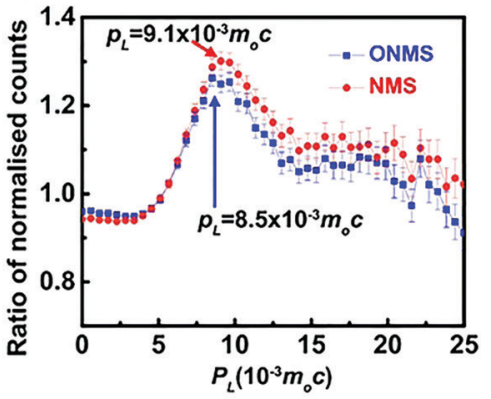

Fig. 4 (a) EPR spectra for ONMS and NMS. (b) Peak normalised positron lifetime spectra for ONMS and NMS. (c) Ratio curves generated from the coincidence Doppler broadening spectra of ONMS and NMS. 
Table 1 Summary of the parameters obtained from positron annihilation lifetime spectroscopy for ONMS and NMS

\begin{tabular}{|c|c|c|c|c|c|c|c|c|c|c|c|c|}
\hline Sample & $\tau_{1}(\mathrm{~ns})$ & $\tau_{2}(\mathrm{~ns})$ & $\tau_{3}(\mathrm{~ns})$ & $I_{1}(\%)$ & $I_{2}(\%)$ & $I_{3}(\%)$ & $\tau_{\mathrm{m}}(\mathrm{ns})$ & $\tau_{\mathrm{b}}(\mathrm{ns})$ & $\tau_{2}-\tau_{\mathrm{b}}(\mathrm{ns})$ & $\kappa\left(\mathrm{ns}^{-1}\right)$ & $\mu\left(\mathrm{ns}^{-1}\right)$ & $C(\mathrm{ppm})$ \\
\hline ONMS & $0.1014 \pm 0.0021$ & $0.2960 \pm 0.0034$ & 0.55 & $34.49 \pm 0.82$ & $52.41 \pm 0.63$ & $13.10 \pm 0.37$ & 0.2621 & 0.1848 & 0.1112 & 3.0891 & 0.6 & 5.148 \\
\hline NMS & $0.1033 \pm 0.0020$ & $0.3047 \pm 0.0029$ & 0.55 & $33.28 \pm 0.72$ & $56.52 \pm 0.55$ & $10.20 \pm 0.32$ & 0.2627 & 0.1900 & 0.1147 & 3.3648 & 0.6 & 5.608 \\
\hline
\end{tabular}

from the positron lifetime results, for the NMS samples is roughly $10 \%$ higher than that of ONMS, which is consistent with the observations of CDBS. Since $\mathrm{O}_{2}$ doping is there in ONMS, the peak of CDBS is slightly shifted towards $P_{\mathrm{L}}=8.5 \times$ $10^{-3} m_{0} c$, which indirectly hints at the annihilation of positrons with the $2 \mathrm{p}$ electrons of $\mathrm{O}_{2}$ atoms $\left(P_{\mathrm{L}}=8.5 \times 10^{-3} m_{0} c\right)$.

The as-grown NMS and ONMS samples along with pristine $\mathrm{MoS}_{2}$ (MS) were employed to study their antifungal properties on fungal pathogens, namely, Alternari aalternata and Fusarium oxysporum and to understand whether doping with elements of different electronegativities affects the fungal inhibition process. The fungitoxicity of the $\mathrm{MoS}_{2}$ nanostructures, as found from our previous study, are highly dependent on their ROS (reactive oxygen species) generation capacity both in dark and under visible light irradiation. ${ }^{37}$ The fungitoxicity of ONMS,
NMS, and MS were determined in terms of their fungal growth inhibition capacity, which was determined by calculating the radial growth reduction in 5 day-old fungal colonies as compared to the control experiment [Fig. S5-S7, ESI $\dagger$ ]. The bare $\mathrm{MoS}_{2}$ (MS) sample, owing to its poor ROS generation capacity, failed to dominate over the fungal ROS scavenging machinery. Hence, no significant fungal inhibition was observed for the bare MS samples. However, doping nitrogen atoms into $\mathrm{MoS}_{2}$ is seen to result in accelerated fungitoxic effects under dark, as revealed by the digital photographs of NMS-treated fungal colonies, which gets further enhanced when treated under visible light irradiation. In the presence of visible light, NMS nanosheets $\left(0.5 \mathrm{mg} \mathrm{mL}{ }^{-1}\right)$ shows $12.5 \%$ radial growth inhibition towards A. alternata, which is 2.12 times greater than that measured under dark experiments [Fig. 5(b)]. However, the
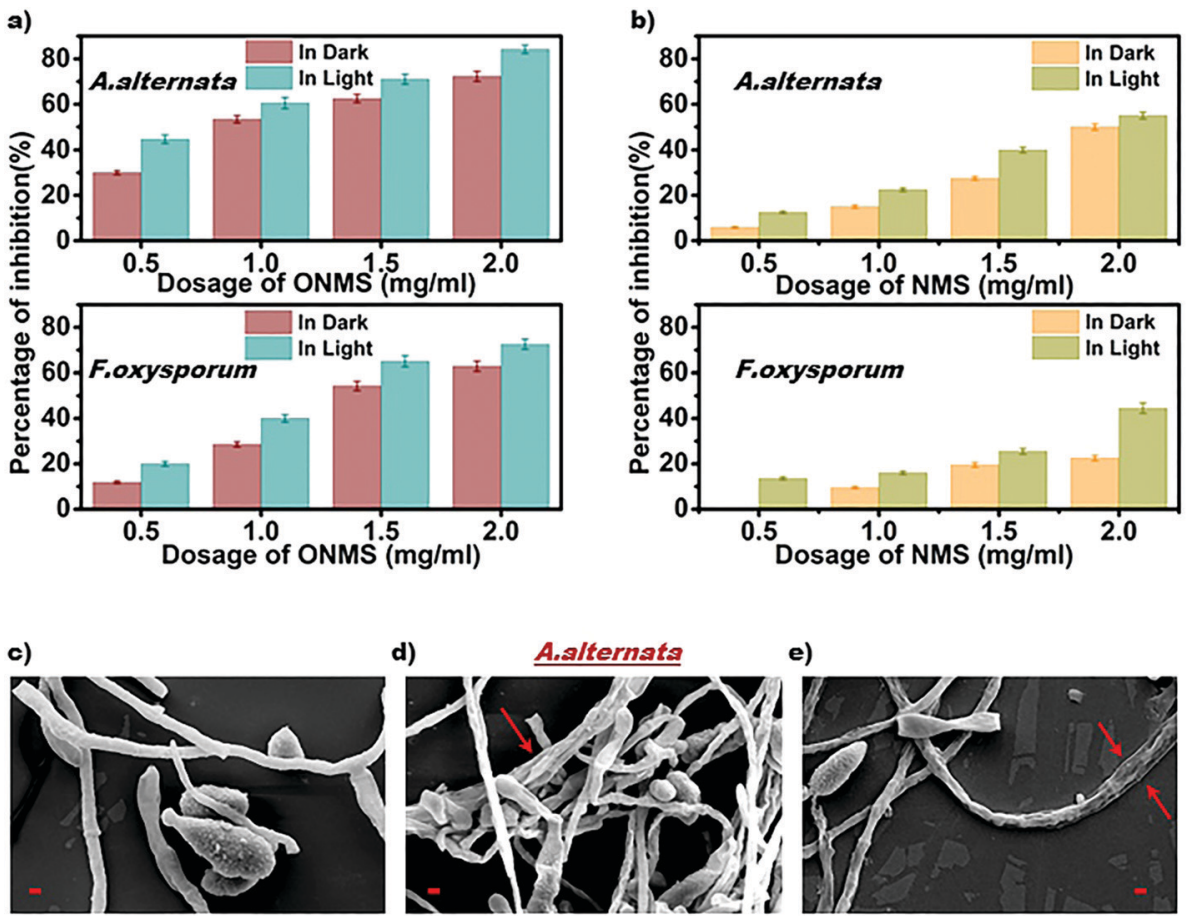

e)
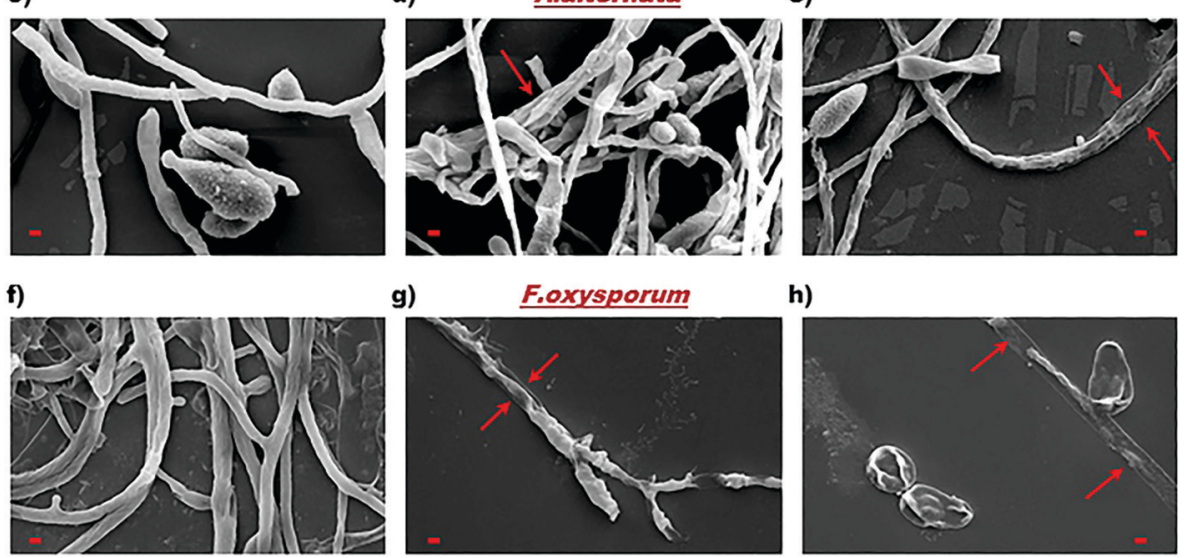

h)

Fig. 5 Graphical representation of (a) ONMS and (b) NMS-induced radial growth inhibition in A. alternata and F. oxysporum. The data represented are statistically significant $(p<0.05$ ); scanning electron micrographs of $A$. alternata and $F$. oxysporum (c and f) without treatment, (d and g) ONMS treatment with a dosage of $2 \mathrm{mg} \mathrm{mL}^{-1}$ in dark, (e and $\mathrm{h}$ ) ONMS treatment with a dosage of $2 \mathrm{mg} \mathrm{mL}^{-1}$ under visible light. All scale bars in red color represent a length of $2 \mu \mathrm{m}$. 
fungitoxicity of the NMS nanostructures increased in a dose dependent manner. Under visible light illumination and for a concentration of $2 \mathrm{mg} \mathrm{mL} \mathrm{mL}^{-1}$, NMS nanostructures showed nearly 55\% mycelial growth retardation in A. alternata, which is 1.1 times higher than the growth retardation achieved with treatment under dark conditions. For F. oxysporum, at lower dosage $\left(0.5 \mathrm{mg} \mathrm{mL}^{-1}\right)$, NMS showed a $12.5 \%$ growth inhibition upon light irradiation, which is very similar to that for A. alternata. However, when treated with $2 \mathrm{mg} \mathrm{mL}^{-1}$ concentration, the fungitoxicity doubles on visible light exposure [Fig. 5(b)]. Higher fungitoxicity in the presence of visible light illumination supports the photo-induced acceleration of ROS generation, resulting in greater cell death. Being a super oxidizing agent, the introduction of oxygen in the $\mathrm{MoS}_{2}$ nanostructure, in addition to nitrogen, accelerated its fungitoxicity further, as expected in the case of ONMS.

Fig. 5(a) shows that for a $2 \mathrm{mg} \mathrm{mL}^{-1}$ concentration and upon visible light treatment, ONMS successfully suppressed $84.21 \%$ mycelial growth of $A$. alternata, which is $16.47 \%$ higher than the retardation obtained under dark experimental conditions. Likewise, under visible light, for $F$. oxysporum, ONMS nanoflowers achieved a fungal growth retardation of $72.54 \%$ with a dosage of $2 \mathrm{mg} \mathrm{mL}^{-1}$, which was $15.41 \%$ higher when treated under dark. Comparing the results obtained for NMS and the ONMS nanoflowers, it is evident that the introduction of oxygen into $\mathrm{MoS}_{2}$ increased their fungitoxicity $\sim 1.5$ and $\sim 1.6$ times for A. alternata and $F$. oxysporum, respectively [Fig. 5(a) and (b)].

The biochemical assessment of fungal cell viability after ONMS treatment were performed by TTC assay. ${ }^{63}$ Both the pathogens were treated with different doses of ONMS nanoflowers for 24 hours, followed by the addition of TTC reagent and further incubation for 12 hours. The dehydrogenase enzymes secreted by viable fungal cells reduced TTC into a red coloured compound, namely, formazan, which was dissolved in dimethyl sulfoxide (DMSO) and detected spectrophotometrically at $570 \mathrm{~nm}$. Cell viability was correlated with formazan formation and is presented in Fig. S8 (ESI $\dagger$ ). In a line similar to the radial growth inhibition assay, ONMS nanoflowers exhibited higher toxicity towards $A$. alternata than $F$. oxysporum, which are $\sim 86 \%$ and $75 \%$, respectively.

Further, the evaluation of the ONMS nanoflower-induced morphological deformities in the fungal cells with reference to the untreated biomass were carried out using an scanning electron microscope and is presented in [Fig. 5(c)-(h)]. For both the pathogens, the smooth surfaced untreated fungal mycelia with a distinct outline gets completely distorted on being treated with doped $\mathrm{MoS}_{2}$ nanoflowers. The release of intra-cellular materials, as evident from the SEM images of the ONMS-treated fungal biomass, is a clear indication of ROS-induced perforations within the cell wall, resulting in its higher permeability, which thereby leads to compulsive cell death. Detection of real-time ROS generation by ONMS nanosheets was performed using DCF dye (dichlorofluorescein) and their effect upon the fungal biomass has been captured under a fluorescence microscope and is presented in Fig. 6. In the case of untreated fungal mycelia, no fluorescence was detected, which indicated the absence of ROS in normal fungal mycelia. In contrast, when treated with ONMS nanoparticles,
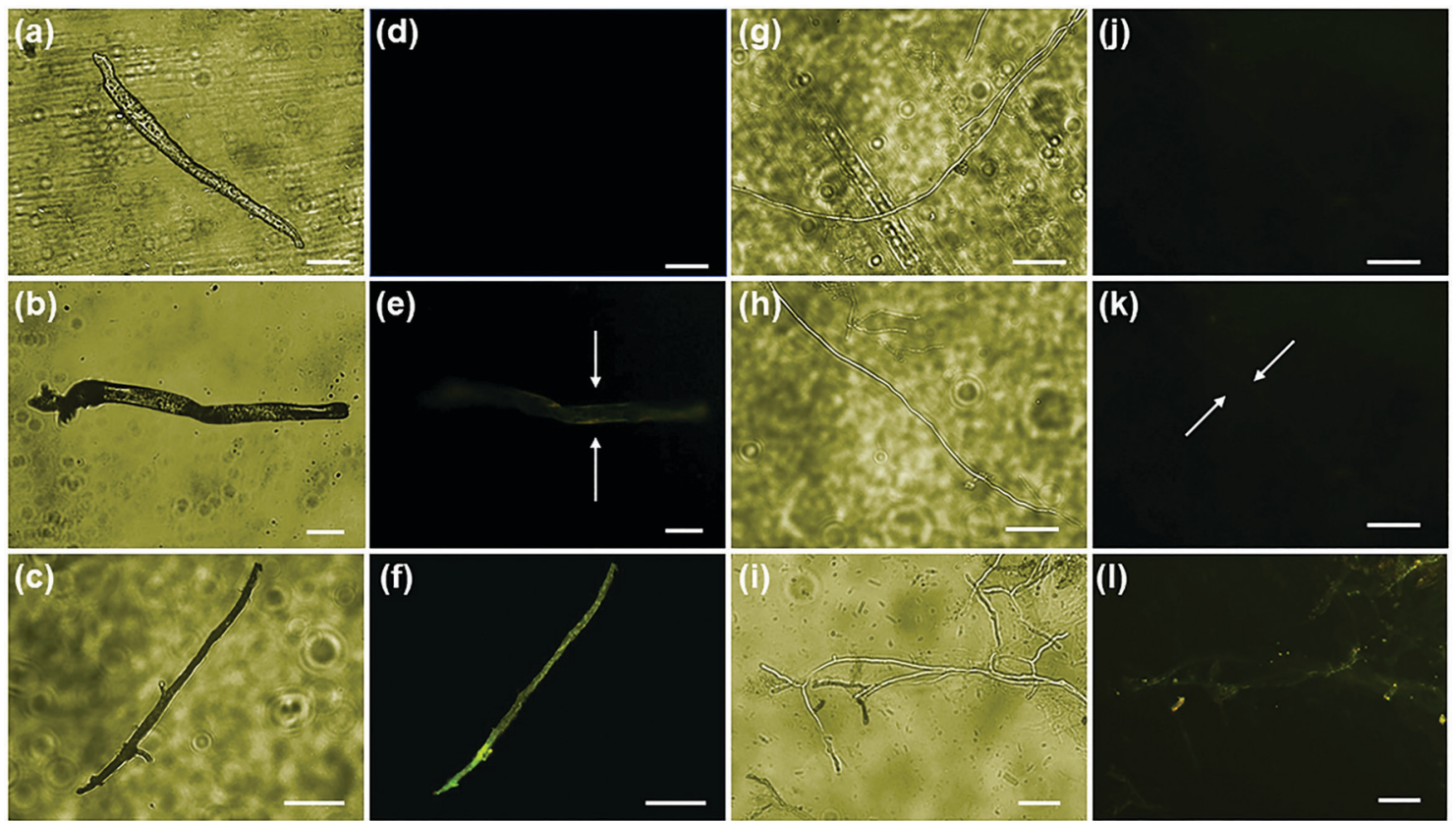

Fig. 6 Fluorescence microscopic imaging of Reactive Oxygen Species (ROS) in ONMS (2 $\mathrm{mg} \mathrm{mL}^{-1}$ ) labelled fungal mycelia. Bright field image of (a-c) A. alternata and ( $\mathrm{g}-\mathrm{i})$ F. oxysporum under various conditions. ( $\mathrm{a}$ and $\mathrm{g}$ ) Without treatment; ( $\mathrm{b}$ and $\mathrm{h}$ ) ONMS treated and grown under dark; (c and i) ONMS treated and grown under visible light irradiation. Fluorescent images of $(\mathrm{d}-\mathrm{f}) \mathrm{A}$. alternata and $(\mathrm{j}-\mathrm{l}) \mathrm{F}$. oxysporum under different conditions. ( $\mathrm{d}$ and $\mathrm{j}$ ) without treatment; (e and k) ONMS treated and grown under dark; ( and l) ONMS treated and grown under visible light irradiation. All scale bars in white represent a length of $50 \mu \mathrm{m}$. 
the emission of green fluorescence was detected upon blue excitation, indicating nanoparticle-induced ROS generation onto the mycelial surface, causing plausible cellular damage and deformities. Again, nanomaterial treatment under visible light illumination shows higher emission of green fluorescence, which supports photo-induced elevation in ROS generation by the nanosheets [Fig. 6].

Nanoparticle-induced fungal cell death has also been confirmed by fluorescence microscopy using EtBr staining (ethidium bromide). The perforated cell wall of the nanoparticletreated fungal biomass allows EtBr binding with DNA material, showing red fluorescence upon green excitation [Fig. 7]. The prominent emission of red fluorescence evident in ONMS and NMS-treated fungal mycelia under visible light irradiation can be attributed as a confirmation of photo-induced accelerated ROS generation [Fig. 6]. However, no red fluorescence was seen in the case of untreated fungal mycelia, which signifies the characteristic rigidity and integrity of the chitin layer, thus successfully eliminating EtBr entry at the intracellular matrix. ROS generation propensity of $\mathrm{MoS}_{2}$ and engineered N-doped $\mathrm{MoS}_{2}$ had been well-documented. ${ }^{30,37}$ The superoxide ${ }^{\bullet} \mathrm{O}_{2}{ }^{-}$and hydroxyl ${ }^{\bullet} \mathrm{OH}$ radicals generated under dark and visible light illumination possess strong oxidation potential. The production of excessive ROS at the cellular environment dominates over its ROS scavenging machinery, thus altering the redox balance at the intracellular level. The impairment in cellular redox-balance imposes the oxidative stress on the cell membrane and affects the membrane permeability severely, leading to compulsive cell death. The generation of superoxide molecules triggers a series of redox reactions inside the cell, synthesizing more toxic molecules that cause oxidation of DNA, lipid, proteins, enzymes, oxidation of water into hydroxyl radical $(\bullet \mathrm{OH})$, and production of toxic $\mathrm{H}_{2} \mathrm{O}_{2}$ molecule. The de-activation of glutathione (GSH) and GSH-related enzymes is one of the most studied toxicological implications of superoxide molecules. GSH plays an important role in the cellular anti-oxidant system. ${ }^{64}$ The oxidation of the -SH group of GSH enzymes leads to stable disulphide (-S-S-) bond formation, resulting in functional inactivation of the enzyme. Consequently, the susceptibility of the fungal cells to the toxic oxidants multiplies several times, exposing it to higher risk of cellular damages. Other than that, the superoxide molecule potentially oxidizes important biomolecules such as DNA, RNA, protein, and lipids, and triggers cellular apoptosis and aging. ${ }^{37,65}$

Oxidation of water molecules generates ${ }^{\bullet} \mathrm{OH}$ radicals that exhibit significant oxidation potential. It strongly reacts with the $\mathrm{H}$ atom of the $\mathrm{N}$-acetyl-glucosamine monomer of the chitin layer and breaks the 1,4 and 1,6-glycosidic linkages, disturbing the characteristic rigidity and integrity of the fungal cell wall. In addition, ${ }^{\bullet} \mathrm{OH}$ radicals also oxidizes intracellular bio-molecules of the microbial cell, thus altering the cellular biochemistry. ${ }^{63,66}$ A schematic representation of the ROS-mediated antifungal mechanism is given in Fig. 8. Superoxide molecules are reduced to $\mathrm{H}_{2} \mathrm{O}_{2}$ and their toxicity to the cell is multi-dimensional. It is actively involved in enhancing cellular apoptosis by DNA fragmentation and chromatin condensation. Higher accumulation of $\mathrm{H}_{2} \mathrm{O}_{2}$ at the cellular level also results in lipid peroxidation and protein oxidation, thus impairing the cellular stability. ${ }^{67}$

The antifungal effect of ONMS was by far better than that of NMS, both in light and in dark. As there is not much significant difference in terms of morphology, vacancy states, and porosity,
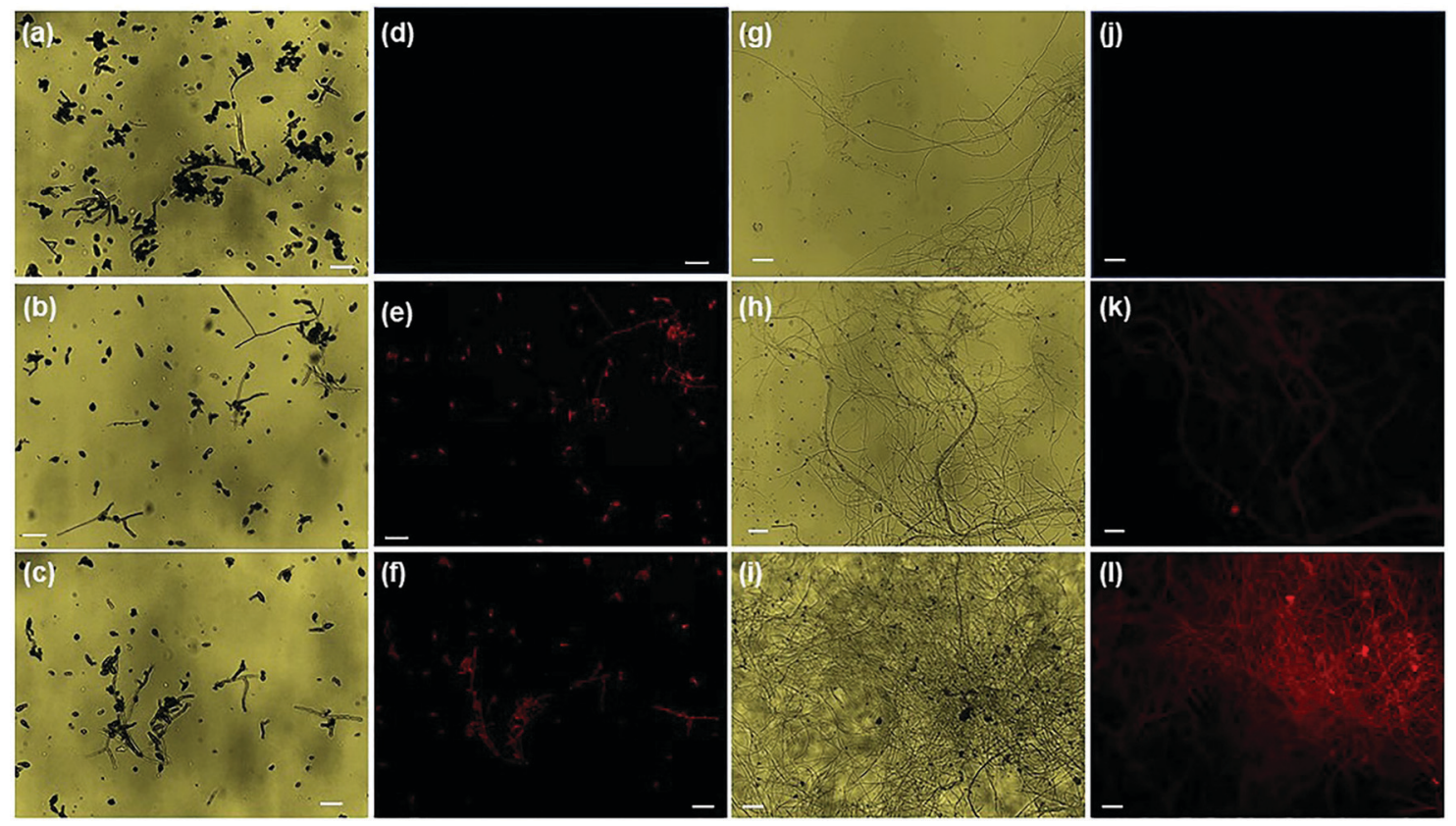

Fig. 7 Fluorescence imaging of fungal DNA by EtBr intercalation through ONMS $\left(2 \mathrm{mg} \mathrm{mL}^{-1}\right)$ treated perforated cell wall. Bright field images of A. alternata and F. oxysporum ( $a$ and $\mathrm{g}$ ) without treatment; ( $\mathrm{b}$ and $\mathrm{h}$ ) treated with ONMS under dark; (c and i) treated with ONMS under visible light irradiation. Fluorescence imaging of $A$. alternata and F. oxysporum ( $\mathrm{d}$ and $\mathrm{j}$ ) without treatment; (e and $\mathrm{k}$ ) treated with ONMS under dark; ( $\mathrm{f}$ and $\mathrm{l}$ ) treated with ONMS under visible light irradiation. All scale bars in white are $50 \mu \mathrm{m}$ in length. 


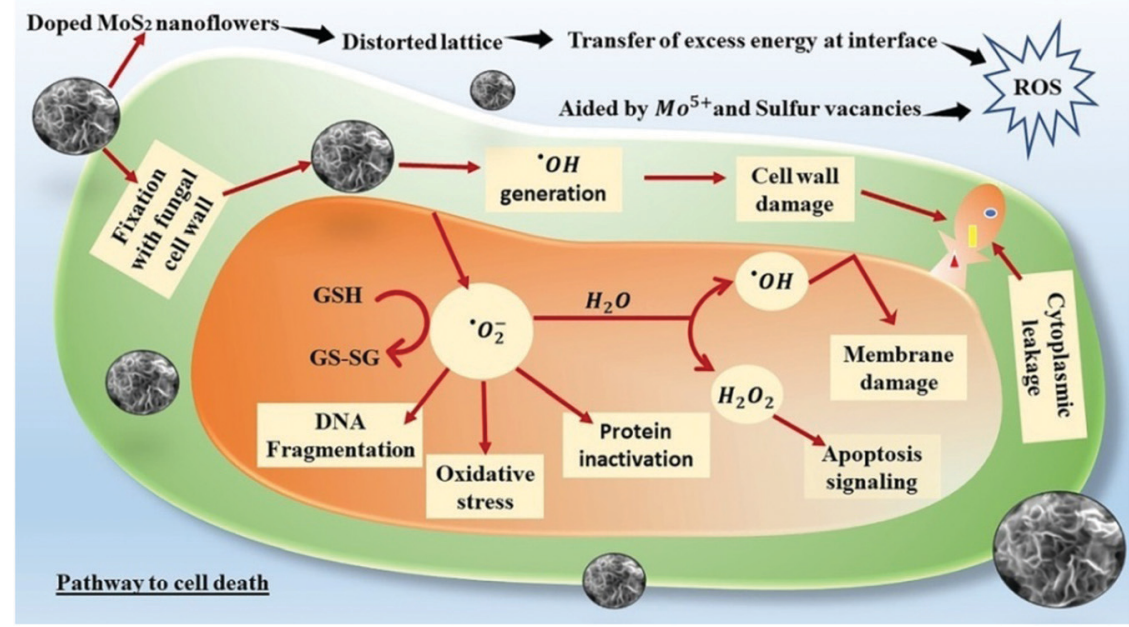

Fig. 8 Schematic representation of the ROS-mediated pathway leading to compulsive cell death.

the better antifungal performance of ONMS in comparison to NMS may be attributed to the elevated levels of ROS generation from the $\mathrm{Mo}^{5+}$ sites in ONMS. Interestingly, unlike our previous work, ${ }^{37}$ in which the S-vacancies played a pivotal role in the spontaneous generation of ROS by N-doped $\mathrm{MoS}_{2}$, ROS generation in the dark in the present study for ONMS was prompted upon by the different mechanism. Because of distortion due to oxygen and nitrogen co-doping, the ONMS lattice is under strain, as revealed from the Raman spectroscopy analysis. On being brought in contact with the aqueous environment (cellular water and constituent water part in the fungal growth media), the strain in doped $\mathrm{MoS}_{2}$ is relaxed and there is a spontaneous transfer of elastic energy stored in the lattice, which thereby triggers the generation of ROS in dark. ROS generation is further augmented under visible light illumination as a result of electron-hole pair generation, which react with dissolved oxygen to form the ROS species. $\mathrm{MoS}_{2}$ being a semiconductor, on being excited with energies greater than its band gap energy, the electrons on its valence band are excited to move to the conduction band, thus creating holes in the $\mathrm{VB} .{ }^{30}$ The better antifungal performance of ONMS originates from the presence of $\mathrm{Mo}^{5+}$ sites in ONMS, as revealed from the EPR studies, which are the active hubs of ROS generation in this case.

The uniqueness and superiority of ONMS over most other nanomaterial-based fungicides lie in the fact that it can generate ROS in dark, which gets enhanced on being illuminated with visible light. Apart from its use in usual antifungal applications, this paves the way for its application in antifungal coatings in implantable medical devices. A comparative study of ONMS with other 2D-nanolayer-based antifungals is given in Table S1 of the ESI, $\dagger$ which shows that the effectiveness towards fungal inhibition of the present sample is in good competition with the presently available fungicides. Further, in order to address the issue of biocompatibility, it may be mentioned here that the constituent elements (Mo and S) of the nanomaterial used in this study are established micro-nutrients that promote plant growth and metabolism. Moreover, bare $\mathrm{MoS}_{2}$, as a compound, does not exhibit any toxicity, which is experimentally evident from our study [Fig. S7, ESI $\dagger$ ]. The toxicity in other samples is purely ROS mediated and achieved by introducing $\mathrm{N}$ and $\mathrm{O}$ into the nanostructure. Further, the $\mathrm{EC}_{50}$ (concentration of $\sim 50 \%$ growth inhibition) value of ONMS for A. alternata and F. oxysporum are 1.0 and $1.5 \mathrm{mg} \mathrm{mL}^{-1}$, respectively. Although, the minute concentration of the nanomaterial is high enough to control the fungal pathogens, the plant's antioxidant system can easily scavenge the ROS generated by the applied nanomaterials. Moreover, there are numerous reports of the excellent biocompatibility of $\mathrm{MoS}_{2}$ and N-doped $\mathrm{MoS}_{2}{ }^{68,69}$ However, to figure out the influence of ONMS on the plant body and to address the issue of biocompatibility, a small experiment was conducted with potato tubers, which ensured the biocompatibility of the present materials towards plant cell. The details of the experiment have been given in the ESI $\dagger$ (Section S1.3).

\section{Conclusions}

In summary, we have successfully incorporated oxygen and nitrogen atoms in $\mathrm{MoS}_{2}$ nanoflowers through an optimised and facile hydrothermal method and studied the antifungal effects of the synthesized samples on fungal pathogens, namely, A. alternata and $F$. oxysporum. The HRTEM, XRD, Raman, XPS, and EPR characterisation results confirmed the presence of O-doping in ONMS. The PAS studies revealed that the overall defect constitution in ONMS and NMS is almost similar with very little differences in the defect history of the two samples. Moreover, doping with oxygen and nitrogen atoms resulted in excellent antifungal activities in $\mathrm{MoS}_{2}$, which otherwise showed no antifungal growth inhibition whatsoever. The antifungal performance of ONMS was superior to that of NMS, both in dark and under visible light illumination. The superiority of ONMS over NMS was achieved through the careful design of the $\mathrm{MoS}_{2}$ nanoflowers through oxygen doping to cultivate the $\mathrm{Mo}^{5+}$ 
states within the lattice. These $\mathrm{Mo}^{5+}$ defect states in ONMS acted as the primary sites for ROS generation, thereby leading to an enhanced performance towards the inhibition of $A$. alternata and $F$. oxysporum. The present study will thus open up the avenue for the design of newer antifungals with lesser chances of antimicrobial resistance through suitable elemental doping in order to cope with the growing threats to global biodiversity due to unprecedented global warming.

\section{Conflicts of interest}

There are no conflicts to declare.

\section{Acknowledgements}

This work was financially supported by the CSIR-India [03(1391)/ 16/EMR-II], UGC-SAP, India [530/20/DRS-II/2015 (SAP-I)] and DST-FIST, India [SR/FST/PSI-219/2016].

\section{References}

1 C. Kinealy, This great calamity: the great Irish Famine: the Irish Famine, Gill \& Macmillan Ltd, 1994, pp. 1845-1852.

2 J. R. Perfect, The antifungal pipeline: a reality check, Nat. Rev. Drug Discovery, 2017, 16(9), 603.

3 C.-Y. Low and C. Rotstein, Emerging fungal infections in immunocompromised patients, F1000Med. Rep., 2011, 3.

4 C. A. Damalas and I. G. Eleftherohorinos, Pesticide exposure, safety issues, and risk assessment indicators, Int. J. Environ. Res. Public Health, 2011, 8(5), 1402-1419.

5 K. Niemirowicz, B. Durnaś, E. Piktel and R. Bucki, Development of antifungal therapies using nanomaterials, Nanomedicine, 2017, 12(15), 1891-1905.

6 A. Panáček, M. Kolář, R. Večeřová, R. Prucek, J. Soukupova, V. Kryštof, P. Hamal, R. Zbořil and L. Kvítek, Antifungal activity of silver nanoparticles against Candida spp, Biomaterials, 2009, 30(31), 6333-6340.

7 A. Karimiyan, H. Najafzadeh, M. Ghorbanpour and S. H. Hekmati-Moghaddam, Antifungal effect of magnesium oxide, zinc oxide, silicon oxide and copper oxide nanoparticles against Candida albicans. Zahedan, J. Res. Med. Sci., 2015, 17(10), 19-23.

8 L. He, Y. Liu, A. Mustapha and M. Lin, Antifungal activity of zinc oxide nanoparticles against Botrytis cinerea and Penicillium expansum, Microbiol. Res., 2011, 166(3), 207-215.

9 S. Zhu, F. Luo, B. Zhu and G.-X. Wang, Toxicological effects of graphene oxide on Saccharomyces cerevisiae, Toxicol. Res., 2017, 6(4), 535-543.

10 M. Sawangphruk, P. Srimuk, P. Chiochan, T. Sangsri and P. Siwayaprahm, Synthesis and antifungal activity of reduced graphene oxide nanosheets, Carbon, 2012, 50(14), 5156-5161.

11 C. Li, X. Wang, F. Chen, C. Zhang, X. Zhi, K. Wang and D. Cui, The antifungal activity of graphene oxide-silver nanocomposites, Biomaterials, 2013, 34(15), 3882-3890.
12 N. Gómez-Ortíz, S. De la Rosa-García, W. González-Gómez, M. Soria-Castro, P. Quintana, G. Oskam and B. OrtegaMorales, Antifungal coatings based on $\mathrm{Ca}(\mathrm{OH})_{2}$ mixed with $\mathrm{ZnO} / \mathrm{TiO}_{2}$ nanomaterials for protection of limestone monuments, ACS Appl. Mater. Interfaces, 2013, 5(5), 1556-1565.

13 N. Meng, N.-L. Zhou, S.-Q. Zhang and J. Shen, Synthesis and antifungal activities of polymer/montmorillonite-terbinafine hydrochloride nanocomposite films, Appl. Clay Sci., 2009, 46(2), 136-140.

14 R. Prucek, J. Tuček, M. Kilianová, A. Panáček, L. Kvítek, J. Filip, M. Kolář, K. Tománková and R. Zbořil, The targeted antibacterial and antifungal properties of magnetic nanocomposite of iron oxide and silver nanoparticles, Biomaterials, 2011, 32(21), 4704-4713.

15 V. Rodríguez-González, R. B. Domínguez-Espíndola, S. CasasFlores, O. A. Patrón-Soberano, R. Camposeco-Solis and S.-W. Lee, Antifungal nanocomposites inspired by titanate nanotubes for complete inactivation of Botrytis cinerea isolated from tomato infection, ACS Appl. Mater. Interfaces, 2016, 8(46), 31625-31637.

16 K.-K. Liu, W. Zhang, Y.-H. Lee, Y.-C. Lin, M.-T. Chang, C.-Y. Su, C.-S. Chang, H. Li, Y. Shi and H. Zhang, Growth of large-area and highly crystalline $\mathrm{MoS}_{2}$ thin layers on insulating substrates, Nano Lett., 2012, 12(3), 1538-1544.

17 Z. Yin, H. Li, H. Li, L. Jiang, Y. Shi, Y. Sun, G. Lu, Q. Zhang, $\mathrm{X}$. Chen and H. Zhang, Single-layer $\mathrm{MoS}_{2}$ phototransistors, ACS Nano, 2012, 6(1), 74-80.

18 Q. Chen, Y. Ouyang, S. Yuan, R. Li and J. Wang, Uniformly wetting deposition of Co atoms on $\mathrm{MoS}_{2}$ monolayer: a promising two-dimensional robust half-metallic ferromagnet, ACS Appl. Mater. Interfaces, 2014, 6(19), 16835-16840.

19 T. Pham, G. Li, E. Bekyarova, M. E. Itkis and A. Mulchandani, $\mathrm{MoS}_{2}$-based optoelectronic gas sensor with sub-parts-per-billion limit of $\mathrm{NO}_{2}$ gas detection, ACS Nano, 2019, 13(3), 3196-3205.

20 L. Zhang, X. Ji, X. Ren, Y. Ma, X. Shi, Z. Tian, A. M. Asiri, L. Chen, B. Tang and X. Sun, Electrochemical ammonia synthesis via nitrogen reduction reaction on a $\mathrm{MoS}_{2}$ catalyst: theoretical and experimental studies, Adv. Mater., 2018, 30(28), 1800191.

21 D. Kufer and G. Konstantatos, Highly sensitive, encapsulated MoS2 photodetector with gate controllable gain and speed, Nano Lett., 2015, 15(11), 7307-7313.

22 Z. Hu, L. Wang, K. Zhang, J. Wang, F. Cheng, Z. Tao and J. Chen, MoS2 nanoflowers with expanded interlayers as high-performance anodes for sodium-ion batteries, Angew. Chem., Int. Ed., 2014, 53(47), 12794-12798.

23 X. Zhu, X. Ji, N. Kong, Y. Chen, M. Mahmoudi, X. Xu, L. Ding, W. Tao, T. Cai and Y. Li, Intracellular mechanistic understanding of 2D $\mathrm{MoS}_{2}$ nanosheets for anti-exocytosisenhanced synergistic cancer therapy, ACS Nano, 2018, 12(3), 2922-2938.

$24 \mathrm{X}$. Li and H. Zhu, Two-dimensional $\mathrm{MoS}_{2}$ : properties, preparation, and applications, J. Materiomics, 2015, 1(1), 33-44.

25 A. Splendiani, L. Sun, Y. Zhang, T. Li, J. Kim, C.-Y. Chim, G. Galli and F. Wang, Emerging photoluminescence in monolayer $\mathrm{MoS}_{2}$, Nano Lett., 2010, 10(4), 1271-1275. 
26 C. Zhu, Z. Zeng, H. Li, F. Li, C. Fan and H. Zhang, Singlelayer $\mathrm{MoS}_{2}$-based nanoprobes for homogeneous detection of biomolecules, J. Am. Chem. Soc., 2013, 135(16), 5998-6001.

27 S. S. Chou, B. Kaehr, J. Kim, B. M. Foley, M. De, P. E. Hopkins, J. Huang, C. J. Brinker and V. P. Dravid, Chemically exfoliated $\mathrm{MoS}_{2}$ as near-infrared photothermal agents, Angew. Chem., Int. Ed., 2013, 52(15), 4160-4164.

28 V. Yadav, S. Roy, P. Singh, Z. Khan and A. Jaiswal, 2D $\mathrm{MoS}_{2^{-}}$ based nanomaterials for therapeutic, bioimaging, and biosensing applications, Small, 2019, 15(1), 1803706.

29 X. Wang, T. Li, H. Ma, D. Zhai, C. Jiang, J. Chang, J. Wang and C. Wu, A 3D-printed scaffold with $\mathrm{MoS}_{2}$ nanosheets for tumor therapy and tissue regeneration, NPG Asia Mater., 2017, 9(4), e376.

30 C. Liu, D. Kong, P.-C. Hsu, H. Yuan, H.-W. Lee, Y. Liu, H. Wang, S. Wang, K. Yan and D. Lin, Rapid water disinfection using vertically aligned $\mathrm{MoS}_{2}$ nanofilms and visible light, Nat. Nanotechnol., 2016, 11(12), 1098-1104.

31 Q. Xu, P. Zhu, J. Zhang, Y. Liu, L. Cai, H. Jiang, M. Ji and J. Chen, Electrochemical formation of distinct nanostructured $\mathrm{MoS}_{2}$ with altered antibacterial activity, Mater. Lett., 2020, 127809.

32 S. Karunakaran, S. Pandit, B. Basu and M. De, Simultaneous exfoliation and functionalization of $2 \mathrm{H}-\mathrm{MoS}_{2}$ by thiolated surfactants: applications in enhanced antibacterial activity, J. Am. Chem. Soc., 2018, 140(39), 12634-12644.

33 S. Begum, A. Pramanik, K. Gates, Y. Gao and P. C. Ray, Antimicrobial Peptide-Conjugated $\mathrm{MoS}_{2}$-Based Nanoplatform for Multimodal Synergistic Inactivation of Superbugs, ACS Appl. Bio Mater., 2018, 2(2), 769-776.

34 X. Zhang, W. Zhang, L. Liu, M. Yang, L. Huang, K. Chen, R. Wang, B. Yang, D. Zhang and J. Wang, Antibiotic-loaded $\mathrm{MoS}_{2}$ nanosheets to combat bacterial resistance via biofilm inhibition, Nanotechnology, 2017, 28(22), 225101.

35 T. I. Kim, B. Kwon, J. Yoon, I.-J. Park, G. S. Bang, Y. Park, Y.-S. Seo and S.-Y. Choi, Antibacterial activities of graphene oxide-molybdenum disulfide nanocomposite films, ACS Appl. Mater. Interfaces, 2017, 9(9), 7908-7917.

36 W. Zhang, Z. Mou, Y. Wang, Y. Chen, E. Yang, F. Guo, D. Sun and W. Wang, Molybdenum disulfide nanosheets loaded with chitosan and silver nanoparticles effective antifungal activities: in vitro and in vivo, Mater. Sci. Eng. Carbon, 2019, 97, 486-497.

37 P. Basu, J. Chakraborty, N. Ganguli, K. Mukherjee, K. Acharya, B. Satpati, S. Khamrui, S. Mandal, D. Banerjee and D. Goswami, Defect-Engineered $\mathrm{MoS}_{2}$ Nanostructures for Reactive Oxygen Species Generation in the Dark: Antipollutant and Antifungal Performances, ACS Appl. Mater. Interfaces, 2019, 11(51), 48179-48191.

38 A. Ramasubramaniam and D. Naveh, Mn-doped monolayer $\mathrm{MoS}_{2}$ : an atomically thin dilute magnetic semiconductor, Phys. Rev. B: Condens. Matter Mater. Phys., 2013, 87(19), 195201.

39 J. Zhou, G. Fang, A. Pan and S. Liang, Oxygen-incorporated $\mathrm{MoS}_{2}$ nanosheets with expanded interlayers for hydrogen evolution reaction and pseudocapacitor applications, ACS Appl. Mater. Interfaces, 2016, 8(49), 33681-33689.
40 J. Xie, J. Zhang, S. Li, F. Grote, X. Zhang, H. Zhang, R. Wang, Y. Lei, B. Pan and Y. Xie, Controllable disorder engineering in oxygen-incorporated $\mathrm{MoS}_{2}$ ultrathin nanosheets for efficient hydrogen evolution, J. Am. Chem. Soc., 2013, 135(47), 17881-17888.

41 T. Sun, Z. Li, X. Liu, L. Ma, J. Wang and S. Yang, Oxygenincorporated $\mathrm{MoS}_{2}$ microspheres with tunable interiors as novel electrode materials for supercapacitors, J. Power Sources, 2017, 352, 135-142.

42 N. M. Brown, N. Cui and A. McKinley, An XPS study of the surface modification of natural $\mathrm{MoS}_{2}$ following treatment in an RF-oxygen plasma, Appl. Surf. Sci., 1998, 134(1-4), 11-21.

43 H. Zhu, X. Qin, L. Cheng, A. Azcatl, J. Kim and R. M. Wallace, Remote plasma oxidation and atomic layer etching of $\mathrm{MoS}_{2}$, ACS Appl. Mater. Interfaces, 2016, 8(29), 19119-19126.

44 J. Martin, J. Cross and L. Pope, $\mathrm{MoS}_{2}$ Interactions with $1.5 \mathrm{eV}$ Atomic Oxygen, MRS Online Proc. Libr., 1988, 140.

45 W. Su, N. Kumar, S. J. Spencer, N. Dai and D. Roy, Transforming bilayer $\mathrm{MoS}_{2}$ into single-layer with strong photoluminescence using UV-ozone oxidation, Nano Res., 2015, 8(12), 3878-3886.

46 J. Wu, H. Li, Z. Yin, H. Li, J. Liu, X. Cao, Q. Zhang and H. Zhang, Layer thinning and etching of mechanically exfoliated $\mathrm{MoS}_{2}$ nanosheets by thermal annealing in air, Small, 2013, 9(19), 3314-3319.

47 C. Lee, H. Yan, L. E. Brus, T. F. Heinz, J. Hone and S. Ryu, Anomalous lattice vibrations of single-and few-layer $\mathrm{MoS}_{2}$, ACS Nano, 2010, 4(5), 2695-2700.

48 A. Azcatl, X. Qin, A. Prakash, C. Zhang, L. Cheng, Q. Wang, N. Lu, M. J. Kim, J. Kim and K. Cho, Covalent nitrogen doping and compressive strain in $\mathrm{MoS}_{2}$ by remote $\mathrm{N}_{2}$ plasma exposure, Nano Lett., 2016, 16(9), 5437-5443.

49 M. Dieterle, G. Weinberg and G. Mestl, Raman spectroscopy of molybdenum oxides Part I. Structural characterization of oxygen defects in $\mathrm{MoO}_{3-x}$ by DR UV/VIS, Raman spectroscopy and X-ray diffraction, Phys. Chem. Chem. Phys., 2002, 4(5), 812-821.

50 J. Xiong, Y. Liu, D. Wang, S. Liang, W. Wu and L. Wu, An efficient cocatalyst of defect-decorated $\mathrm{MoS}_{2}$ ultrathin nanoplates for the promotion of photocatalytic hydrogen evolution over CdS nanocrystal, J. Mater. Chem. A, 2015, 3(24), 12631-12635.

51 M. Nagai, A. Irisawa and S. Omi, XPS Study of the Deactivation and Sulfiding of Nitrided Molybdena-Alumina Catalyst during the Hydrodesulfurization of Dibenzothiophene. The, J. Phys. Chem. B, 1998, 102(39), 7619-7626.

52 B. J. Tan, K. J. Klabunde and P. M. Sherwood, XPS studies of solvated metal atom dispersed (SMAD) catalysts. Evidence for layered cobalt-manganese particles on alumina and silica, J. Am. Chem. Soc., 1991, 113(3), 855-861.

53 S. Knipe, J. Mycroft, A. Pratt, H. Nesbitt and G. Bancroff, X-ray photoelectron spectroscopic study of water adsorption on iron sulphide minerals, Geochim. Cosmochim. Acta, 1995, 59(6), 1079-1090.

54 C. Zhang, J. Sunarso and S. Liu, Designing $\mathrm{CO}_{2}$-resistant oxygen-selective mixed ionic-electronic conducting membranes: 
guidelines, recent advances, and forward directions, Chem. Soc. Rev., 2017, 46(10), 2941-3005.

55 S. J. Hilsenbeck, V. G. Young Jr and R. E. McCarley, Synthesis, structure, and characterization of $\mathrm{N}$-ligated $\mathrm{Mo}_{6} \mathrm{~S}_{8} \mathrm{~L}_{6}$ cluster complexes. Molecular precursors to chevrel phases, Inorg. Chem., 1994, 33(9), 1822-1832.

56 L. Benoist, D. Gonbeau, G. Pfister-Guillouzo, E. Schmidt, G. Meunier and A. Levasseur, X-ray photoelectron spectroscopy characterization of amorphous molybdenum oxysulfide thin films, Thin Solid Films, 1995, 258(1-2), 110-114.

57 T. A. Patterson, J. C. Carver, D. E. Leyden and D. M. Hercules, A surface study of cobalt-molybdena-alumina catalysts using X-ray photoelectron spectroscopy, J. Phys. Chem., 1976, 80(15), 1700-1708.

58 S. McDonnell, A. Azcatl, R. Addou, C. Gong, C. Battaglia, S. Chuang, K. Cho, A. Javey and R. M. Wallace, Hole contacts on transition metal dichalcogenides: interface chemistry and band alignments, ACS Nano, 2014, 8(6), 6265-6272.

59 J. Kibsgaard, Z. Chen, B. N. Reinecke and T. F. Jaramillo, Engineering the surface structure of $\mathrm{MoS}_{2}$ to preferentially expose active edge sites for electrocatalysis, Nat. Mater., 2012, 11(11), 963-969.

60 J. R. González, R. Alcántara, J. L. Tirado, A. J. Fielding and R. A. Dryfe, Electrochemical interaction of few-layer molybdenum disulfide composites $v s$. sodium: new insights on the reaction mechanism, Chem. Mater., 2017, 29(14), 5886-5895.

61 C. Louis and M. Che, EPR investigation of the coordination sphere of molybdenum $(5+)$ ions on thermally reduced silica-supported molybdenum catalysts prepared by the grafting method, J. Phys. Chem., 1987, 91(11), 2875-2883.
62 R. Nieminen and J. Laakkonen, Positron trapping rate into vacancy clusters, Appl. Phys., 1979, 20(2), 181-184.

63 S. S. Boxi, K. Mukherjee and S. Paria, Ag doped hollow $\mathrm{TiO}_{2}$ nanoparticles as an effective green fungicide against Fusarium solani and Venturia inaequalis phytopathogens, Nanotechnology, 2016, 27(8), 085103.

64 X. Yang, J. Li, T. Liang, C. Ma, Y. Zhang, H. Chen, N. Hanagata, H. Su and M. Xu, Antibacterial activity of twodimensional $\mathrm{MoS}_{2}$ sheets, Nanoscale, 2014, 6(17), 10126-10133.

65 N. R. Panyala, E. M. Peña-Méndez and J. Havel, Silver or silver nanoparticles: a hazardous threat to the environment and human health?, J. Appl. Biomedicine, 2008, $6,3$.

66 K. Mukherjee, K. Acharya, A. Biswas and N. R. Jana, $\mathrm{TiO}_{2}$ Nanoparticles Co-doped with Nitrogen and Fluorine as Visible-Light-Activated Antifungal Agents, ACS Appl. Nano Mater., 2020, 3(2), 2016-2025.

67 M. Kawai-Yamada, Y. Ohori and H. Uchimiya, Dissection of Arabidopsis Bax inhibitor-1 suppressing Bax-, hydrogen peroxide-, and salicylic acid-induced cell death, The Plant Cell, 2004, 16(1), 21-32.

68 J. H. Appel, D. O. Li, J. D. Podlevsky, A. Debnath, A. A. Green, Q. H. Wang and J. Chae, Low cytotoxicity and genotoxicity of two-dimensional $\mathrm{MoS}_{2}$ and $\mathrm{WS}_{2}$, ACS Biomater. Sci. Eng., 2016, 2(3), 361-367.

69 F.-Y. Wu, Y.-S. Cheng, D.-M. Wang, M.-L. Li, W.-S. Lu, X.-Y. Xu, X.-H. Zhou and X.-W. Wei, Nitrogen-doped $\mathrm{MoS}_{2}$ quantum dots: Facile synthesis and application for the assay of hematin in human blood, Mater. Sci. Eng. Carbon, 2020, 110898. 